\title{
Viral non-coding RNA inhibits HNF4a expression in HCV associated hepatocellular carcinoma
}

\author{
Zhao Wang ${ }^{1}$, Kristin Ceniccola², Liliana Florea ${ }^{3}$, Bi-Dar Wang ${ }^{2}$, Norman H. Lee² and Ajit Kumar ${ }^{* *}$
}

\begin{abstract}
Background: Hepatitis C virus (HCV) infection is an established cause of chronic hepatitis, cirrhosis and hepatocellular carcinoma (HCC); however, it is unclear if the virus plays a direct role in the development of HCC. Hepatocyte nuclear factor $4 a$ (HNF4a) is critical determinant of epithelial architecture and hepatic development; depletion of HNF4a is correlated with oncogenic transformation. We explored the viral role in the inhibition of HNF4a expression, and consequent induction of tumor-promoting genes in HCV infection-associated HCC.

Methods: Western blot analysis was used to monitor the changes in expression levels of oncogenic proteins in liver tissues from HCV-infected humanized mice. The mechanism of HNF4a depletion was studied in HCV-infected human hepatocyte cultures in vitro. Targeting of HNF4a expression by viral non-coding RNA was examined by inhibition of Luciferase HNF4a 3'-UTR reporter. Modulation of invasive properties of HCV-infected cells was examined by Matrigel cell migration assay.

Results: Results show inhibition of HNF4a expression by targeting of HNF4a 3'-UTR by HCV-derived small non-coding RNA, vmr11. Vmr11 enhances the invasive properties of HCV-infected cells. Loss of HNF4a in HCV-infected liver tumors of humanized mice correlates with the induction of epithelial to mesenchymal transition (EMT) genes.

Conclusions: We show depletion of HNF4a in liver tumors of HCV-infected humanized mice by HCV derived small non-coding RNA (Vmr11) and resultant induction of EMT genes, which are critical determinants of tumor progression. These results suggest a direct viral role in the development of hepatocellular carcinoma.
\end{abstract}

\section{Background}

Hepatitis $\mathrm{C}$ virus $(\mathrm{HCV})$ infection is a major cause of chronic hepatitis, liver cirrhosis, and hepatocellular carcinoma (HCC) [1]. Each year over a half million new cases of HCC are diagnosed worldwide, with approximately 20,000 new cases in the United States alone; $\mathrm{HCC}$ related to $\mathrm{HCV}$ infection is the most rapidly rising cause of cancer-related deaths in the United States [2]. However, patients who are treated and achieve a sustained virologic response have a markedly reduced risk of developing HCC. We examined the possible role of viral factors in the development of HCC using MUP$\mathrm{uPA} / \mathrm{SCID} / \mathrm{Bg}$ mice engrafted with human hepatocytes and infected with HCV [3]. We describe results showing

\footnotetext{
* Correspondence: akumar@gwu.edu

'Department of Biochemistry, The George Washington University,

Washington, DC, USA

Full list of author information is available at the end of the article
}

viral non-coding RNA vmr11 directed loss of $H N F 4 \alpha$ and subsequent induction of EMT genes in HCVinfected HCC in the humanized mouse model. We used human primary hepatocyte cultures [4] to study the mechanism of HNF4 $\alpha$ depletion by the HCV-derived small non-coding RNA.

Hepatocyte nuclear factor $4 \alpha(\mathrm{HNF} 4 \alpha)$ is member of the steroid hormone receptor superfamily of nuclear transcription factors with critical roles in hepatocyte differentiation, liver development and the maintenance of epithelial architecture [5, 6]. Liver-specific inactivation of HNF $4 \alpha$ is linked to hepatomegaly, abnormal deposition of glycogen, cholesterol and lipid metabolism [7-9]. HNF4 $\alpha$ contributes to the regulation of a large fraction of liver transcriptome by binding to promoters of actively transcribed genes [10]. HNF4 $\alpha$ plays a critical role in the maintenance of hepatic epithelium by suppressing expression of epithelial to mesenchymal transition (EMT) 
genes [11]. Loss of HNF4 $\alpha$ has been shown to result in the induction of EMT genes and oncogenic transformation [11-13]. We sought to understand the mechanism of HNF4 $\alpha$ depletion and regulation of tumor development by investigating whether $\mathrm{HCV}$ triggers the loss of HNF4 $\alpha$ and consequent induction of tumorigenic genes in HCC.

A normal level of HNF4 $\alpha$ protein is critical in maintaining hepatic epithelial architecture and liver function. Depletion of HNF4 $\alpha$ is a critical determinant of epithelialto-mesenchymal transition, characterized by loss of the epithelial marker E-Cadherin and induction of the mesenchymal marker Vimentin during tumor progression. Here we describe results showing that loss of HNF4 $\alpha$ is triggered by HCV-derived 22 nucleotide vmr11 RNA [14]. Vmr11 RNA is sufficient to confer HCV-infected hepatocytes with invasive properties. Sustained loss of HNF4 $\alpha$ in $\mathrm{HCV}$ infection-associated liver tumor down-regulated $E$ Cadherin and induced the expression of Vimentin and EMT regulatory genes Snail, TGF- $\beta$ and HMGA2. These results support a direct viral role of $\mathrm{HCV}$ in the development of hepatocellular carcinoma by the targeting of HNF4 $\alpha$ expression with viral non-coding RNA, vmr11.

\section{Methods}

\section{Transfection of PPH cultures with HCV1a genomic RNA}

$\mathrm{HCV}$ genomic RNA was prepared by linearizing pCVH77c (HCV genotype 1a cDNA) plasmid with Xba1, followed by run-off transcription with T7 Ribomax Express (Promega). $5 \times 10^{5}$ human primary hepatocytes $(\mathrm{PPH})$ were transfected with $1 \mu \mathrm{g}$ of the H77 RNA using Fugene 6 (Promega) procedure [14]. Vmr11 oligonucleotides transfections were carried out using Lipofectamine ${ }^{\odot}$ 2000 Transfection Reagent (Invitrogen ${ }^{\mathrm{Tm}}$ ), with 50nM vmiR-11 mimic or, 50nM vmiR-11 (LNA-modified) antagomir. The vmiR-11 oligonucleotides were transfected twice at 24-h intervals and the cells were harvest at $48 \mathrm{~h}$ post-transfection.

\section{RNA extraction}

Total RNA from human hepatocyte cultures or the liver tissues were lysed by TRIzol ${ }^{\circ}$ Reagent (Ambion) according to the manufacturer's protocol. Briefly, tissue samples were cut into small piece and rinsed in $1 \mathrm{~mL}$ of TRIzol $^{\circ}$ Reagent. Liver tissues were homogenized and total RNA was separated with $0.2 \mathrm{~mL}$ chloroform and precipitated with $0.5 \mathrm{~mL}$ Iso-propanol. The RNA pellet was washed with $75 \%$ ethanol. RNA concentration was measured using the NanoDrop 1000 Spectrophotometer.

\section{Reverse transcription and quantitative real time PCR}

First strand cDNA was synthesized by QuantiTect Reverse Transcription protocol (QIAGEN) with 1 microgram RNA samples. The cDNA products were diluted
50 fold for qRT-PCR assay. RT-PCR was performed using iTaq Universal SYBR Green Supermix with Rox (Bio-Rad) on ABI 7300 Real Time PCR system. Primers used were as follows: $H N F 4 \alpha$-forward: 5'-TGTCC CGACA GATCA CCTC- 3'; HNF4 $\alpha$-reverse: 5'- CACTC AACGA GAACC AGCAG- 3'; 18 s rRNA-forward: 5'- GTAAC CCGTT GAACC CCATT -3', 18 s rRNA-reverse: 5' -CCATC CAATC GGTAG TAGCG -3'. Virus replication was monitored either by estimating genomic equivalents (GE) of HCV RNA (by nested PCR [4]) from virus particles recovered from the culture medium, or by immune blotting for viral antigen, NS5A or HCV core [4].

\section{Protein extraction and immunoblotting}

PPH cultures (treated as indicated) were lysed with RIPA buffer supplemented with $1 \mathrm{X}$ protease inhibitor cocktail (Roche). $10 \mu \mathrm{g}$ of protein was analyzed on $10 \%$ precast Mini-PROTEIN gel (BIO-RAD). Proteins were transferred to PVDF membrane, blocked with $5 \%$ non-fat dry milk and probed with antibodies as indicated. HRP conjugated anti-rabbit, anti-mouse, anti-goat antibodies (from Abcam; SuperSignal West Dura Chemiluminescent Substrate) were visualized under BIO-RAD ChemiDoc $^{\mathrm{Tm}}$ XRS + System. Images were analyzed (by Image Lab $^{\text {th }}$ Software developed by BIO-RAD), and the protein amounts estimated by normalizing to $\beta$-Actin as internal control [14].

Liver tissues used for Western blot analysis were from human hepatocyte engrafted MUP-uPA/SCID/Bg mice, either uninfected (controls) or $\mathrm{HCV}$ infection-induced liver tumors. The chimeric mouse liver tissues for these studies were provided by Drs. Tesfaye and Feinstone [3]. The liver tissues were lysed with RIPA buffer supplemented with protease inhibitor cocktail (Roche). $10 \mu \mathrm{g}$ of protein was analyzed on $10 \%$ precast Mini-PROTEIN gel (BIO-RAD). The gels were transferred to PVDF membrane, blocked with $5 \%$ non-fat dry milk and probed with antibodies (Abcam) against the proteins as indicated. HRP conjugated anti-rabbit, or anti-goat antibodies (Abcam; SuperSignal West Dura Chemiluminescent Substrate) were visualized using BIO-RAD ChemiDoc $^{\text {Tm }}$ XRS + System. Western blots were analyzed by Image Lab $^{\text {mix }}$ Softwar developed by BIO-RAD; and the protein quantitation was based on $\beta$-Actin internal (loading) control.

\section{Matrigel assay}

Primary human hepatocytes $\left(1 \times 10^{4}\right)$ were seeded in the top well of a Matrigel-coated invasion chamber (BD Biosciences) in DMEM containing $0.1 \%$ serum. The bottom well was filled with $750 \mu \mathrm{L}$ DMEM containing $10 \%$ serum as a chemoattractant. After $48 \mathrm{~h}$, non-invading cells were scraped from the upper side of the insert using a cotton swab. Invading cells on the bottom of the 
insert were fixed and stained with Diff-Quick Stain (IMEB, Inc., San Marcos, CA) according to manufacturer's instructions. The total number of invading cells was counted for each insert under a light microscope.

\section{Results}

\section{Loss of HNF4a in HCV-infection associated hepatocellular} carcinoma

We attempted to establish a link between HCV infection and loss of HNF4 $\alpha$ in HCV infection associated HCC using a recently reported [3] chimeric mouse model. Earlier studies with transgenic mice explored a direct role of $\mathrm{HCV}$ proteins in promoting hepatocarcinogenesis $[15,16]$. Transgenic mice however, do not model HCV infection associated liver cancer, nor are the tumors of human origin.

Mercer et al [17] reported successful HCV infection in chimeric mice that had much of their livers replaced by human liver. These immunodeficient (SCID/bg) mice carry tandem copies of urokinase plasminogen activator (uPA) gene under the control of albumin promoter (Alb-uPA). In an improved version of uPA transgenic mouse, the tandem array of uPA genes was put under the control of major urinary protein (MUP) promoter [18]; MUP is not expressed until after three weeks of life. The MUP-uPA mice were crossed with SCID/bg mice and triply homozygous, MUP-uPA/SCID/bg mice were recovered; these mice are robust, breed well with high infant survival, and can be transplanted with human hepatocytes and infected with (most genotypes of) $\mathrm{HCV}$ [3]. The control animals in this study were engrafted but not infected with $\mathrm{HCV}$, and remained healthy for up to eleven months.

Representative Western blots of HNF4 $\alpha$ protein in liver tissues of three tumor- bearing and three control mice are shown in Fig. 1a. Quantitative assessment of $\mathrm{HNF} 4 \alpha$ protein in $\mathrm{HCV}$-infected liver tumors was made from similar Western blot analyses of seven controls and eight tumor tissues from human hepatocyte-engrafted and HCV-infected MUP-uPA/SCID/Bg mice (Fig. 1b). The results suggest nearly three-fold depletion of $\mathrm{HNF} 4 \alpha$ protein in $\mathrm{HCV}$-infected liver tumors.

\section{Depletion of HNF4a leads to the induction of EMT markers}

An important consequence of HNF4 $\alpha$ depletion is the erosion of normal epithelial architecture and acquisition of mesenchymal markers, a hallmark of tumor progression $[6,19,20]$. We investigated the consequence of $\mathrm{HCV}$ infection-associated loss of HNF4 $\alpha$ during development of liver tumors of humanized mice. HNF4 $\alpha$ normally functions to maintain hepatic epithelial architecture by inhibiting the expression of epithelial to

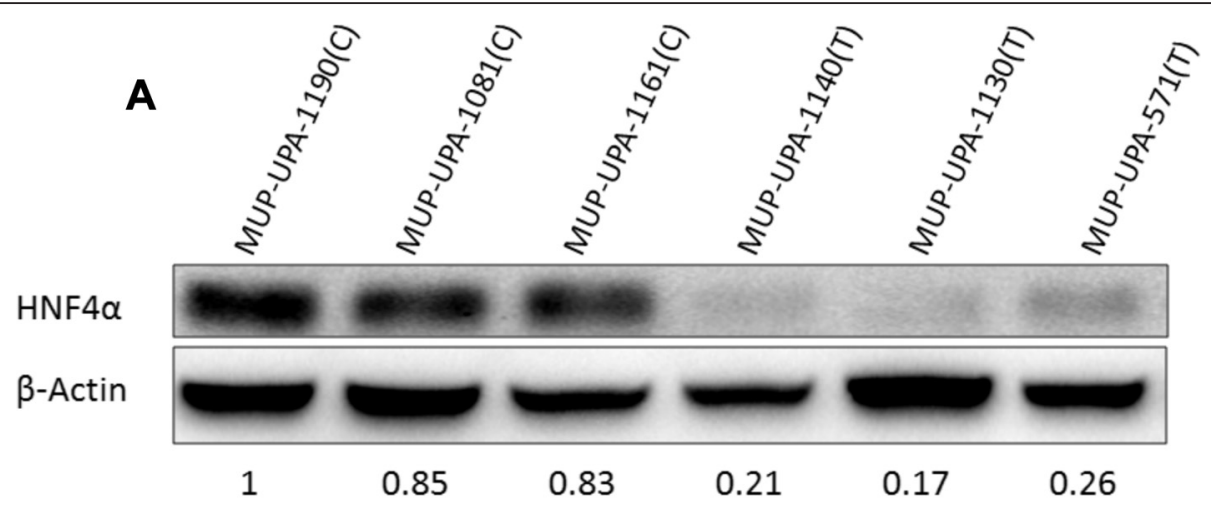

B

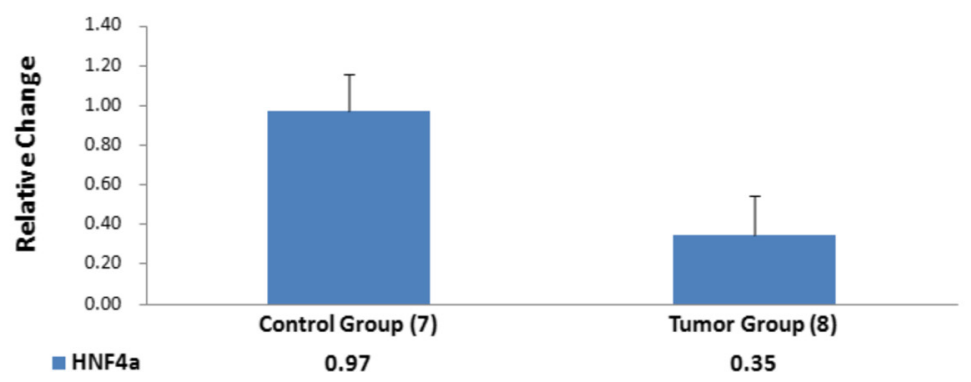

Fig. 1 HNF4a protein in HCV-infection associated liver tumor: (a). Representative Western blots of three controls (c), and three liver tumors (T) are shown. Change in HNF4a a protein level was normalized to $\beta$-Actin loading control; relative values are indicated underneath each lane. b Quantitative change in HNF4a protein levels was estimated by similar Western blot analyses of 7 controls and 8 liver tumors from HCV infected chimeric mice. The relative values of HNF4a shown were based on Western blots run in triplicates (mean ${ }^{+/}$SE) $\left({ }^{*} p<0.01\right)$ 
mesenchymal transition (EMT) genes. By contrast, loss of HNF4 $\alpha$ promotes the expression of EMT genes and tumor progression. An earlier report described induction of EMT genes in HCV-infected human primary hepatocytes [21]. We describe results that build on these studies and show that induction of EMT in HCV-infected human hepatocytes is a direct consequence of targeting of $\mathrm{HNF} 4 \alpha$ 3'-UTR by HCV-derived vmr11 RNA.

Genes associated with epithelial-mesenchymal transition are critical determinants of tumor progression.
Among the EMT master regulators, Snail is a strong repressor of E-cadherin, leading to the loss of epithelial structure and enhanced invasive properties of the tumor [12]. Vimentin by contrast is predominantly expressed in mesenchymal cells. We observed a marked induction of Vimentin in HCV-infected liver tumors compared to control liver tissues of human hepatocyte-engrafted but uninfected MUP-uPA/SCID/Bg mice (Fig. 2a).

HNF4 $\alpha$ is known to suppress the transcription of EMT master regulatory genes, Snail, Slug and HMGA2
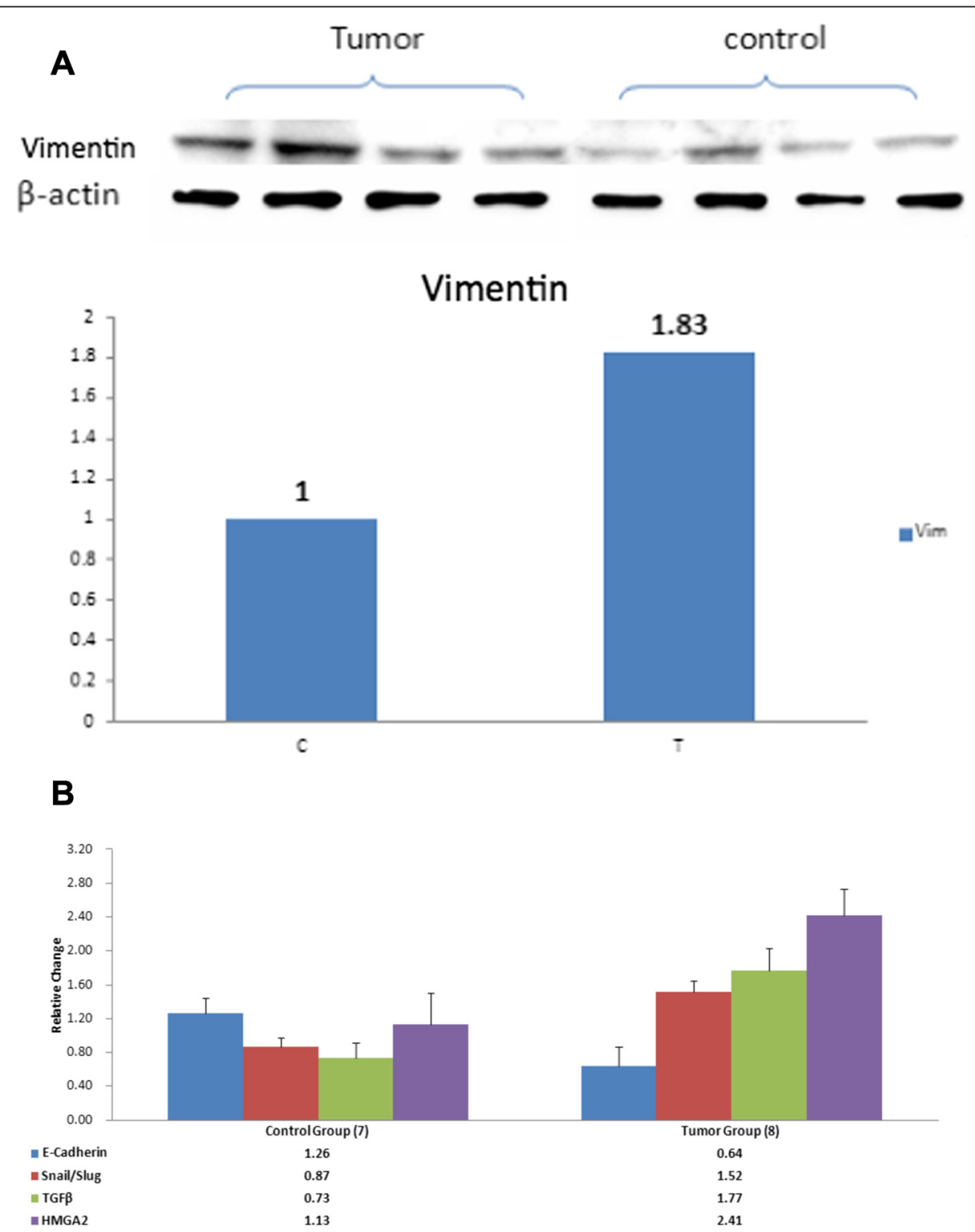

Fig. 2 Altered expression of Vimentin and EMT markers in liver tumors of HCV-infected MUP-UPA/SCID/Bg mice: (2a) Induction of Vimentin in liver tumor was compared to the liver tissue from human hepatocyte engrafted but uninfected chimeric mice. Representative Western blots of four tumors and four control liver is shown in the upper panel with $\beta$-Actin loading control. Lower panel shows relative increase in Vimentin in liver tumors was compared to the uninfected controls. (2b) Expression levels of EMT regulatory genes in liver tumors. Liver protein from seven uninfected controls and eight HCV infected humanized mice were compared by Western blotting (run in triplicate); Relative values (Mean +/- SE, normalized to $\beta$-Actin loading control) of each protein is given underneath 
[11]. Snail family of zinc finger transcription repressors regulate epithelial to mesenchymal transition (EMT) [22], partly through direct inhibition of E-cadherin [12, 23]. We investigated whether HCV infection-related loss of $\mathrm{HNF} 4 \alpha$ promotes coordinated induction of EMT genes in human hepatocyte-engrafted HCV-infected SCID/Bg mice. Results (Fig. 2b) suggest that depletion of HNF4 $\alpha$ in HCV-infected liver tumors correlates with the induction of Snail, HMGA2 and TGF- $\beta$ and the suppression of E-cadherin expression.

\section{Viral non-coding RNA, vmr11 targets HNF4a 3'-UTR}

We explored the mechanism of HNF4 $\alpha$ depletion in $\mathrm{HCV}$-infected human hepatocytes. In an earlier report we described HCV-derived small non-coding RNA, vmr11 that displayed protooncogenic properties by blocking nuclear translocation of PTEN protein, and inducing $\gamma$ H2AX, a marker of DNA double strand breaks [14]. Bioinformatics search of vmr11 targets based on the 'PITA' program [24] identified potential vmr11 target sequence within HNF4a 3'-UTR.
To assess a possible direct role of HCV-derived vmr11 in the depletion of $\mathrm{HNF} 4 \alpha$, we first determined the effects of vmr11 on HNF4 $\alpha$ depletion in human hepatocytes transfected with HCV genomic RNA. Results (shown in Fig. 3) indicate that the loss of HNF4 $\alpha$ protein in cells transfected with HCV genomic RNA is largely restored by the introduction of antisense vmr11 oligonucleotides. Interestingly, we observed similar decline in HNF $4 \alpha$ protein levels of cells transfected with vmr11 oligonucleotides alone. Competition of the effects of vmr11 RNA in vivo by co-transfection of wild-type vmr11 and antisense vmr11 oligonucleotides restored HNF4 $\alpha$ protein levels to that of mock-transfected control cells (Fig. 4). The results suggest that the loss of HNF4 $\alpha$ protein, either in HCV replicating cells (transfected with HCV genomic RNA), or in cells transduced with vmr11 oligonucleotides alone, is due to the effects of vmr11 RNA. The results suggest that HCV-derived vmr11 RNA contributes to the loss of HNF4 $\alpha$ protein in $\mathrm{HCV}$ infected human hepatocytes.

We then investigated whether the decline in HNF4 $\alpha$ protein, either due to $\mathrm{HCV}$ replication or by artificially

A

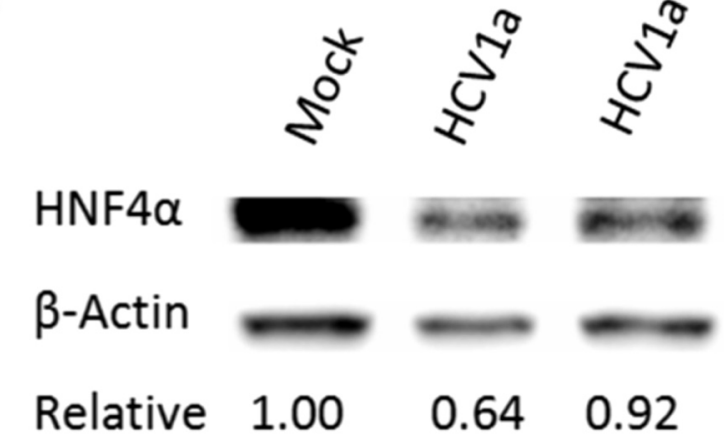

B

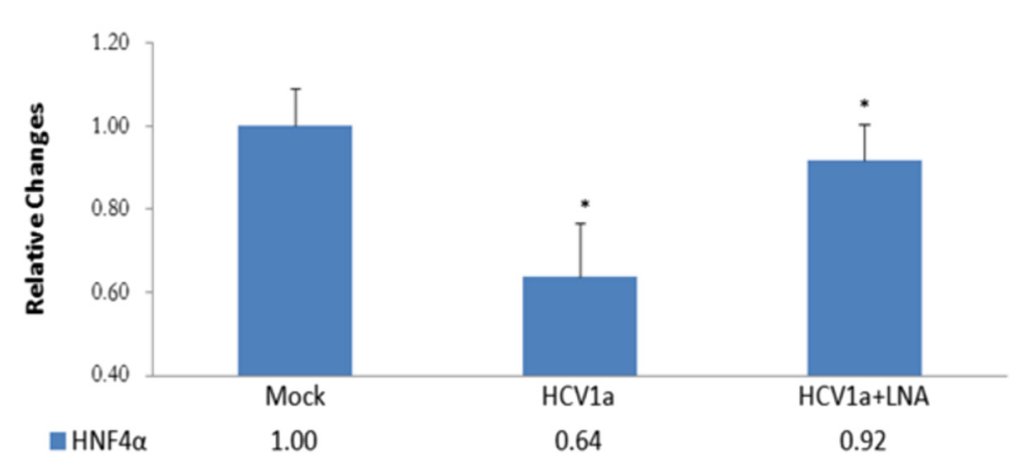

Fig. 3 Inhibition of HNF4a in human hepatocytes transfected with HCV genomic RNA. Upper part (a) shows Western blots of HNF4a (from left to right), from mock transfected control cells, cells transfected with $1 \mu \mathrm{g} \mathrm{HCV} \mathrm{(H77)} \mathrm{genomic} \mathrm{RNA} \mathrm{and} \mathrm{(far} \mathrm{right} \mathrm{lane),} \mathrm{cells} \mathrm{transfected} \mathrm{with} 1 \mu \mathrm{g}$ HCV RNA plus 50nM LNA-vmR-11antagomir. Cells were harvested $48 \mathrm{~h}$ post transfection. Lower part (b) shows relative change in HNF4a protein levels as compared to the mock-transfected cells (analyzed in triplicate Mean $=/-\mathrm{SE},{ }^{*} p<0.03$ ) 
A

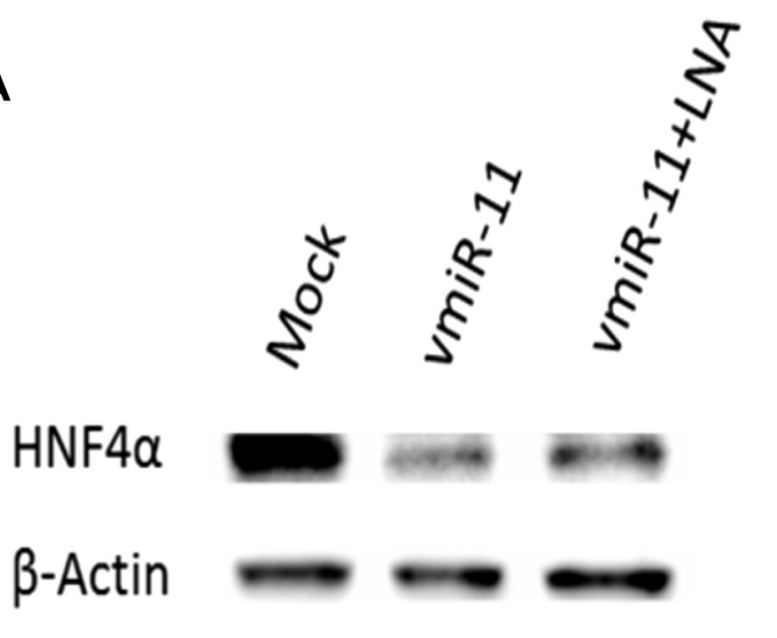

\section{Relative $\quad \begin{array}{lll}1.00 & 0.60 & 0.82\end{array}$}

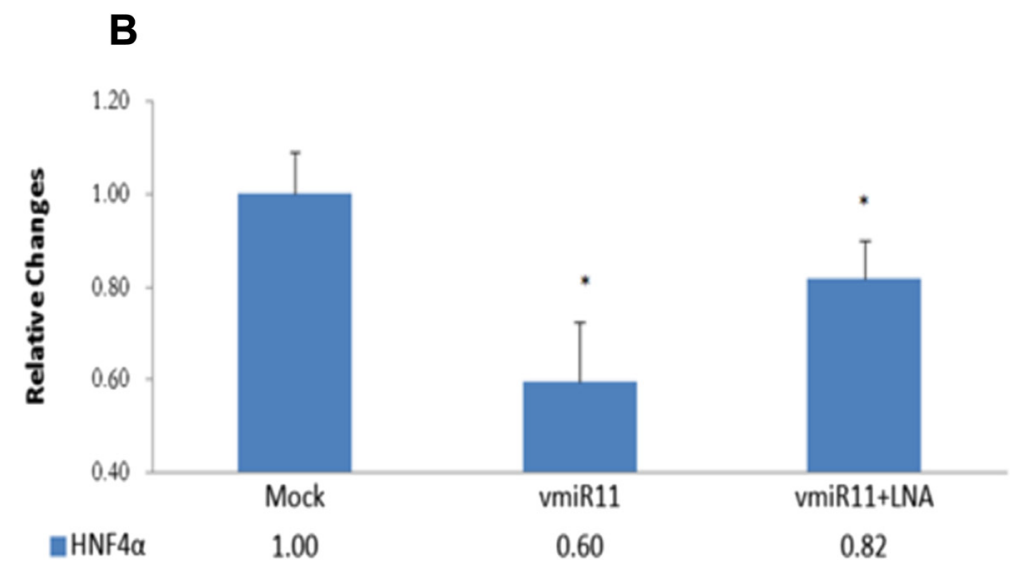

Fig. 4 Inhibition of HNF4a by vmr11: Upper part (a), shows Western blot of HNF4a from control (mock transfected) cells, cells transfected with 50nM vmir-11 "mimic" oligonucleotides, and (far right lane), cells transfected with 50nM vmir-11 mimic plus 50nM vmir-11 antagomir. Transfections with vmr11 oligonucleotides were repeated at 24-h intervals. Cells were harvested at $48 \mathrm{~h}$. Lower part (b), shows relative changes in HNF 4 a protein levels normalized to $\beta$-Actin loading control $\left.{ }^{*} p<0.03\right)$

increasing intracellular vmr11 RNA, could result from the loss of HNF4 $\alpha$ mRNA. We determined $H N F 4 \alpha$ mRNA levels in cells transfected with HCV genomic RNA or vmr11 oligonucleotides by RT-PCR. Results of HNF $4 \alpha$ mRNA analysis (Fig. 5) indicate no significant loss of HNF4 $\alpha$ mRNA, either in cells transfected with HCV genomic RNA or vmr11 oligonucleotides. This suggests that the loss of HNF4 $\alpha$ in HCV-infected cells is initiated by post-transcriptional silencing of $\mathrm{HNF} 4 \alpha$ by vial non-coding RNA, vmr11.

We further investigated whether the predicted vmr11 target site within HNF4 $\alpha$ mRNA 3'-UTR is recognized by vmr11 RNA to block HNF4 $\alpha$ expression. To do this, we determined the extent of inhibition of Luciferase-
HNF4 $\alpha$-3'UTR reporter gene expression with increasing amounts of vmr11 oligonucleotides. To ascertain that vmr11-directed inhibition of HNF4 $\alpha$ is not due to the instability of vmr11 oligonucleotides introduced into the cells, we used equimolar amounts of standard vmr11 oligonucleotides and 2'-Fluoro-stabilized vmr11 RNA. We co-transfected Luciferase HNF4 $\alpha$ 3'-UTR reporter plasmid [13] with increasing amounts of either the wild-type vmr11 ("mimic") oligunucleotides, or 2'-Fluoro-modified vmr11 oligonucleotides (TriLink BioTechnolgies). Luciferase reporter assays suggest that introduction of either normal vmr11 oligonucleotides, or 2'-Fluoro-stabilized vmr11 RNA was equally efficient in blocking HNF4 $\alpha$ expression; about $75 \%$ down regulation of $\mathrm{HNF} 4 \alpha$ protein 

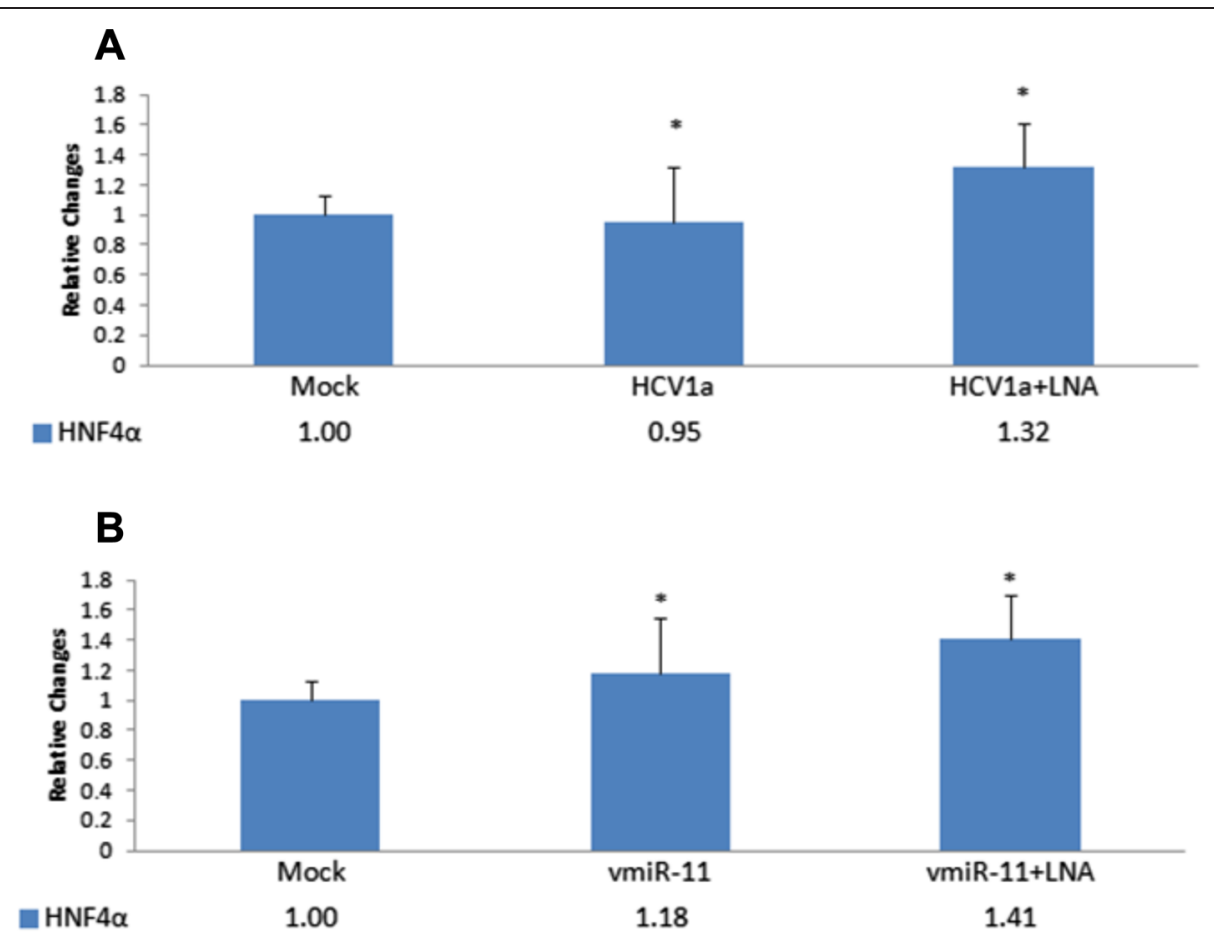

Fig. 5 HNF4a mRNA levels in HCV RNA or vmr11 transfected cells. Upper panel (a): RT-PCR analysis of HNF4a mRNA from mock transfected, HCV (H77) genomic RNA transfected or HCV (H77) genomic RNA plus vmiR-11 antagomir co-transfected cells; Lower panel (b); RT-PCR analysis of HNF4a mRNA from mock transfected cells, or cells transfected with wild-type vmr11 ("mimic") oligonucleotides or vmiR-11 mimic oligonucleotides plus LNA-vmr11 antagomir. The transfection conditions were as described in Fig. 4. Relative values (SEM) of HNF4a mRNA were estimated from three independent RT-PCR runs $\left({ }^{*} p<0.01\right)$

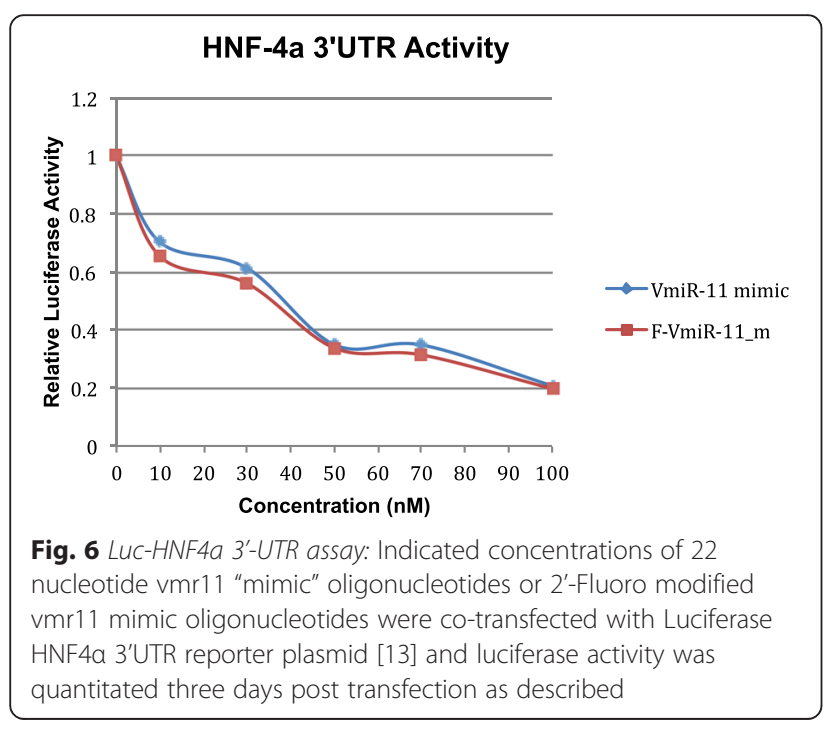

was observed, compared to cells transfected with irrelevant, scrambled oligonucleotides (Fig. 6).

\section{Enhanced invasive properties of human hepatocytes} transfected either with HCV genomic RNA or vmr11 oligonucleotides, can be counteracted by antisense vmr11 Results suggesting that HCV-derived vmr11 RNA inhibited $\mathrm{HNF} 4 \alpha$ expression, prompted us to ask if depletion of HNF4 $\alpha$ is sufficient to promote oncogenic changes in HCV-infected human hepatocytes. We addressed the issue by Matrigel cell invasion assay by comparing acquired cell invasive properties of human hepatocytes transfected either with HCV genomic RNA (H77), or the 22 nt vmr11 "mimic" RNA (F-vmr11-m), (Fig. 7). Introduction of either HCV genomic RNA or vmr11 oligonucleotides resulted in significant enhancement of cell migration as compared to cells transfected with irrelevant, scrambled oligonucleotides. The cell-invasive properties induced either by HCV genomic RNA or vmr11 "mimic" oligonucleotides were reversed by introduction of antivmr11 oligonucleotides, suggesting that $\mathrm{HCV}$-derived vmr11 RNA is responsible for promoting cell invasive properties of HCV-infected cells. 


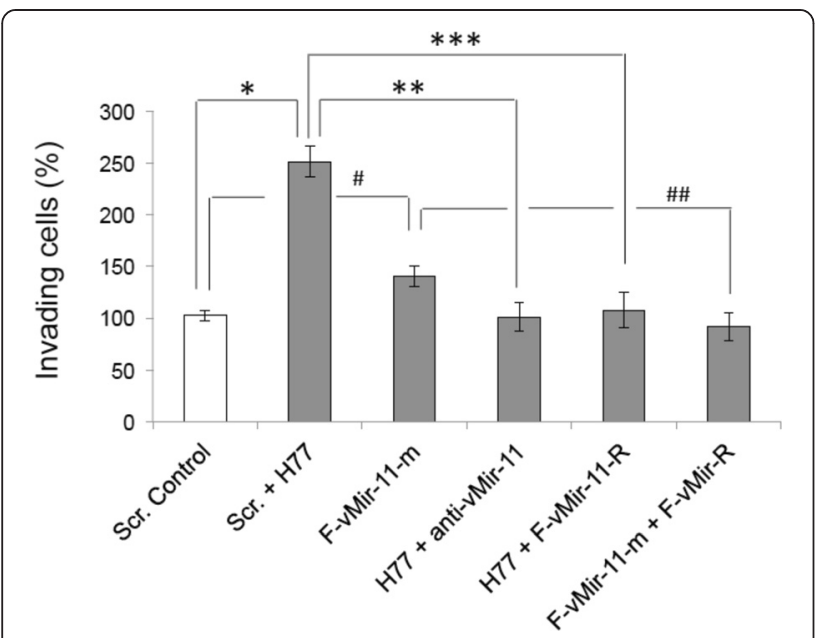

Fig. 7 Matrigel cell invasion assay: (From left to right): Hepatocytes transfected with 50nM scrambled (Scr.) nonspecific control oligonucleotides, Scrambled plus H77 (HCV1a) genomic RNA, 2'-Fluoro modified vmr11 mimic (F-vMir-11-m), HCV 1a genomic RNA (H77) plus anti-vmr11 (anti-vMir-11), H77 plus 2'-Fluoro anti-vmr11 (F-vmr11-R), and Fluoro-vmr11-mimic plus Fluoro-vmr11-R. Three days posttransfection, the cells were processed for Matrigel assay. Data are mean + S.E.M. from 6 independent experiments for each bar graph. *Significantly different from Scr. Control by ANOVA with post-hoc Dunnett's test $(P<0.05)$

\section{Discussion}

In a recent report [14] we described a negative strand $\mathrm{HCV}$ genomic RNA-derived small non-coding RNA (vmr11) and its ability to post-transcriptionally silence Transportin 2 (TRN2) expression. Transportin 2 interacts with PTEN tumor suppressor; TRN2-PTEN proteinprotein interaction is required for nuclear translocation of PTEN. Significantly, nuclear insufficiency of PTEN in $\mathrm{HCV}$-infected cells resulted in induction of $\gamma \mathrm{H} 2 \mathrm{AX}$, a marker of DNA double strand break and genomic instability [14]. Such oncogenic properties of vmr11-regulated genes prompted us to investigate whether vmr11 target genes include other tumor suppressors that would be critical in promoting $\mathrm{HCV}$ infection-associated HCC. Bioinformatics search for vmr11 targets [24] suggested HNF4 $\alpha$ tumor suppressor as a potential target of regulation by the viral non-coding RNA.

The results described here show post-transcriptional silencing of HNF $4 \alpha$ by vmr11, and suggest an intriguing possibility that viral non-coding RNA initiated loss of $\mathrm{HNF} 4 \alpha$ is sustained in HCV-infected humanized mice to promote HCC. The self-reinforcing circuit of hepatocellular transformation as reported in recent studies [13], indicated that siRNA-mediated transient suppression of HNF $4 \alpha$ is sustained by establishing a miRNA feed-back loop without genetic mutation [25]. Here we explored the mechanism that triggers of HNF4 $\alpha$ depletion in vivo. The experiments described here investigate whether the suppression of HNF4 $\alpha$ and consequent induction of
$\mathrm{HCV}$ infection-associated liver tumor development is regulated by viral non-coding RNA. We examined the loss of HNF $4 \alpha$ in vivo in a humanized mouse model of $\mathrm{HCV}$ infection-associated HCC. We explored the mechanism of direct viral role in the onset of HNF4 $\alpha$ depletion in HCV-infected human hepatocyte cultures.

Epithelial Cadherin (E-Cadherin) is the central target of snail and slug (also known as SNAI1 and SNAI2) transcriptional regulators. Epithelial cells express ECadherin, whereas mesenchymal cells express Vimentin [26]. During normal development and organogenesis epithelial to mesenchymal transition (EMT) is achieved by integrating a complex series of extracellular signals that are transient [19]. In tumor development the EMTpromoting stimuli are sustained by tumorigenic factors. The inhibition of E-Cadherin and induction of Vimentin are considered established markers of EMT and tumor progression. Our results suggest that progression of $\mathrm{HCV}$ infection-associated $\mathrm{HCC}$ is sustained by down regulation of HNF4 $\alpha$ and consequent induction of EMT promoting snail/slug, negative transcriptional regulators of E-cadherin. EMT is also triggered by soluble growth factor such as TGF- $\beta$ induced in parallel with snail/slug transcription regulators in liver tumors of $\mathrm{HCV}$-infected chimeric mice.

The high-mobility group protein 2 (HMGA2) is a nonhistone chromatin protein that is primarily expressed in tumors of mesenchymal origin. HMGA2 plays particularly important role in EMT maintenance of metastatic human lung and pancreatic cancer. Our results showing increased levels of HMGA2 in HCV infection-associated HCC of humanized mice are consistent with role of HMGA2 in the maintenance of EMT and liver tumor progression. Transforming growth factor- $\beta$ (TGF- $\beta$ is known to regulate the expression of EMT genes in cooperation with HMGA2 [27]. Our results showing coordinate induction of HMGA2, TGF- $\beta$ along with the EMT markers are consistent with the model that viral non-coding RNA targeted depletion of HNF4 $\alpha$ sets the stage for the development of $\mathrm{HCC}$, tumor invasion and metastasis. Acquisition of a mesenchymal phenotype may allow HCV-infected cells to invade surrounding stroma and disseminate proliferative foci through the liver. These studies suggest the feasibility of testing antisense RNAdirected therapy of HCV infection-associated HCC.

Results described here suggest that epigenetic regulation of hepatocellular transformation is triggered by the inhibition of HNF4alpha, a direct taget of HCV-derived small non-coding RNA [14]. Our in vitro experiments with human hepatocyte cultures suggest targeting of HNF4 $\alpha$ 3'-UTR by vmr11 RNA as the initiating event of HNF $4 \alpha$ depletion in HCV-infected cells. Depletion of HNF4 $\alpha$ in HCV infection-associated hepatocellular carcinoma promotes EMT genes and tumor progression. 
Targeting of HNF4 $\alpha$ by viral small non-coding RNA more closely links $\mathrm{HCV}$ infection to hepatocellular transformation, and raises the possibility of antisense RNA-based therapy of $\mathrm{HCV}$ infection-associated liver cancer.

\section{Competing interests}

Although Alere supplied the ELISA and LAM strip-tests free of charge they had no role in the design and conduct of the study, analysis of the data or writing of the manuscript. The authors have no other interests to declare.

\section{Authors' contribution}

ZW, KC and B-D W performed the biochemical experiments. LF performed the bioinformatics analysis of vmr11 targeting. NHL an AK wrote the paper. All authors read and approved the final manuscript.

\section{Acknowledgement}

We thank Dr. Maria Hatziapostolou (ULA) for the Lucifersae HNF4a 3'UTR plasmid, and Heiwa Nakazima Foundation for support. The research work in Dr. Kumar's lab was supported by Katzen Cancer Research Foundation of The George Washington University.

\section{Author details}

'Department of Biochemistry, The George Washington University, Washington, DC, USA. ${ }^{2}$ Department of Pharmacology, and Program in Molecular Oncology, The George Washington University, Washington, DC, USA. ${ }^{3}$ McKusick-Nathans Institute of Genetic Medicine, The Johns Hopkins University, Baltimore, MD, USA.

Received: 7 March 2015 Accepted: 22 June 2015

Published online: 08 July 2015

\section{References}

1. El-Serag HB, Rudolph KL. Hepatocellular carcinoma: epidemiology and molecular carcinogenesis. Gastroenterology. 2007;132(7):2557-76.

2. El-Serag HB. Hepatocellular carcinoma. N Engl J Med. 2011:365(12):1118-27.

3. Tesfaye A, Stift J, Maric D, Cui Q, Dienes HP, Feinstone SM. Chimeric mouse model for the infection of hepatitis B and C viruses. PLoS One. 2013;8(10), e77298.

4. Banaudha K, Orenstein J, Korolnek T, St Laurent G, Wakita 3rd T, Kumar A. Primary hepatocyte culture supports hepatitis $C$ virus replication: a model for infection-associated hepatocarcinogenesis. Hepatology. 2010;51(6):1922-32.

5. Z Zhong W, Sladek FM, Darnell Jr JE. The expression pattern of a Drosophila homolog to the mouse transcription factor HNF-4 suggests a determinative role in gut formation. The EMBO J. 1993;12(2):537-44.

6. Parviz F, Matullo C, Garrison WD, Savatski L, Adamson JW, Ning G, et al. Hepatocyte nuclear factor 4alpha controls the development of a hepatic epithelium and liver morphogenesis. Nat Genet. 2003;34(3):292-6.

7. Hayhurst GP, Lee YH, Lambert G, Ward JM, Gonzalez FJ. Hepatocyte nuclear factor 4alpha (nuclear receptor 2A1) is essential for maintenance of hepatic gene expression and lipid homeostasis. Mol Cell Biol. 2001;21(4):1393-403.

8. Shih DQ, Bussen M, Sehayek E, Ananthanarayanan M, Shneider BL, Suchy FJ, et al. Hepatocyte nuclear factor-1alpha is an essential regulator of bile acid and plasma cholesterol metabolism. Nat Genet. 2001;27(4):375-82

9. Martinez-Jimenez CP, Kyrmizi I, Cardot P, Gonzalez FJ, Talianidis I. Hepatocyte nuclear factor 4alpha coordinates a transcription factor network regulating hepatic fatty acid metabolism. Mol Cell Biol. 2010;30(3):565-77.

10. Odom DT, Zizlsperger N, Gordon DB, Bell GW, Rinaldi NJ, Murray HL, et al. Control of pancreas and liver gene expression by HNF transcription factors. Science. 2004;303(5662):1378-81.

11. Santangelo L, Marchetti A, Cicchini C, Conigliaro A, Conti B, Mancone C, et al. The stable repression of mesenchymal program is required for hepatocyte identity: a novel role for hepatocyte nuclear factor 4alpha. Hepatology. 2011;53(6):2063-74.

12. Cano A, Perez-Moreno MA, Rodrigo I, Locascio A, Blanco MJ, del Barrio MG, et al. The transcription factor snail controls epithelial-mesenchymal transitions by repressing E-cadherin expression. Nat Cell Biol. 2000;2(2):76-83.

13. Hatziapostolou M, Polytarchou C, Aggelidou E, Drakaki A, Poultsides GA, Jaeger SA, et al. An HNF4alpha-miRNA inflammatory feedback circuit regulates hepatocellular oncogenesis. Cell. 2011;147(6):1233-47.
14. Bao W, Florea L, Wu N, Wang Z, Banaudha K, Qian J, et al. Loss of nuclear PTEN in HCV-infected human hepatocytes. Infect Agents Cancer. 2014;9:23.

15. McGivern DR, Lemon SM. Virus-specific mechanisms of carcinogenesis in hepatitis C virus associated liver cancer. Oncogene. 2011;30(17):1969-83.

16. Moriya K, Fujie H, Shintani Y, Yotsuyanagi H, Tsutsumi T, Ishibashi K, et al. The core protein of hepatitis $C$ virus induces hepatocellular carcinoma in transgenic mice. Nat Med. 1998;4(9):1065-7.

17. Mercer DF, Schiller DE, Elliott JF, Douglas DN, Hao C, Rinfret A, et al. Hepatitis $C$ virus replication in mice with chimeric human livers. Nat Med. 2001;7(8):927-33.

18. Braun KM, Sandgren EP. Cellular origin of regenerating parenchyma in a mouse model of severe hepatic injury. Am J Pathol. 2000;157(2):561-9.

19. Thiery JP, Sleeman JP. Complex networks orchestrate epithelialmesenchymal transitions. Nat Rev Mol Cell Biol. 2006;7(2):131-42.

20. Kalluri $R$, Weinberg RA. The basics of epithelial-mesenchymal transition. J Clin Invest. 2009;119(6):1420-8.

21. Bose SK, Meyer K, Di Bisceglie AM, Ray RB, Ray R. Hepatitis C virus induces epithelial-mesenchymal transition in primary human hepatocytes. J Virol. 2012;86(24):13621-8.

22. Carver EA, Jiang R, Lan Y, Oram KF, Gridley T. The mouse snail gene encodes a key regulator of the epithelial-mesenchymal transition. Mol Cell Biol. 2001;21(23):8184-8.

23. Hajra KM, Chen DY, Fearon ER. The SLUG zinc-finger protein represses Ecadherin in breast cancer. Cancer Res. 2002;62(6):1613-8.

24. Kertesz M, lovino N, Unnerstall U, Gaul U, Segal E. The role of site accessibility in microRNA target recognition. Nat Genet. 2007;39(10):1278-84.

25. Schwabe RF, Wang TC. Targeting liver cancer: first steps toward a miRacle? Cancer Cell. 2011;20(6):698-9.

26. Peinado $\mathrm{H}$, Portillo F, Cano A. Transcriptional regulation of cadherins during development and carcinogenesis. Int J Dev Biol. 2004;48(5-6):365-75.

27. Huber MA, Kraut N, Beug H. Molecular requirements for epithelialmesenchymal transition during tumor progression. Curr Opin Cell Biol. 2005;17(5):548-58

\section{Submit your next manuscript to BioMed Central and take full advantage of:}

- Convenient online submission

- Thorough peer review

- No space constraints or color figure charges

- Immediate publication on acceptance

- Inclusion in PubMed, CAS, Scopus and Google Scholar

- Research which is freely available for redistribution

Submit your manuscript at www.biomedcentral.com/submit 